\title{
AI, Robotics, and the Workplace of the Future
}

\author{
Artyom Kravchenko
}

\begin{abstract}
Robotics has been developing at an enormous speed with the AI advancements of the past decades. Today's robots boast advanced performance, dexterity, and ever-increasing intelligence that allow them to analyze the dynamic external environment and employ contingent decision-making. While machine learning is still at the germinal stage of development, some notable steps forward (e.g., probabilistic modelling, Bayesian optimization, data compression) have been made to equip contemporary robots with more functionality. Such improvements contributed to the commonplace introduction of automatons in the workplace to substitute human labor, thus causing rising concerns about the threats of industrial computerization to the human jobs globally. This article explores the implementation of AI in robotics, determines the ways in which intelligent robots change the contemporary workplace, and lays out the forthcoming changes expected in the job structure and skills related to workplace robotization.
\end{abstract}

\section{Introduction}

The humanity has already been fascinated with an idea to create rational, intelligent robots. Since the advent of primitive mechanical assistants in the Industrial Age, people have been working on improving their design and functionality. Though this area of technological development still remains ambiguous in terms of machine ethics and artificial agents' morality, application of artificial intelligence (AI) in robotics has aided the industry enormously (Ashrafian, 2015). AI and robotics have traditionally shared the goal of designing and building intelligent agents that perceive reason about, and act upon, the everyday world. Thus, today's machines don't function only as a human being's "mechanical arm"; they can perform complex multi-step tasks and operate under computer control without human intervention.

AI has contributed to the modern advancements of robotics in many ways. It has improved the robots' perception and reasoning about physical objects in terms of space, path planning, dealing with uncertainty, and compliance with complex human instructions (Brady, 1985). In simple terms, AI has enabled machines to learn from their experience, which is presently implemented in the industrial practice via probabilistic modelling. The latter has grown into one of the most common theoretical and practical approaches to designing machines capable of experiential learning. The probabilistic framework has also enabled dealing with uncertainty, which is the most critical challenge of machine learning. The AI- 
enriched robotics thus witnessed considerable progress in the form of innovative, state-of-theart technologies such as probabilistic programming, Bayesian optimization, data compression, and automatic model discovery (Ghahramani, 2015).

The AI-led advances in robotics are an indisputable step forward in a variety of industries as they perform some of the human functions more precisely, quickly, and efficiently. Moreover, they enable exploration of dangerous sites such as depths of the ocean and space - the places which people cannot reach due to physical limitations. However, the rapid technological progress and fast advancements in robotics have caused heightened concerns among workers of all specialties in terms of the potential danger to their employment. There is a common opinion suggesting that up to one-third of today's jobs will be taken by robots by 2025 (Frey \& Osborne, 2013; Lynch, 2015). This article thus explores the overall connection of AI and robotics in detail, examines the changes that AI-enriched robotics is gradually introducing in the modern workplace, and discusses the potential implications of those changes for workers.

\section{Defining AI and Robotics}

Artificial intelligence is defined as a branch of science aiding machines to find solutions to complex problems and deal with uncertainty in a human-like manner, which implies equipping machines with human features such as intelligence and ability to learn (Tirgul \& Naik, 2016). Due to these purposes, AI commonly works on the advancements of math, psychology, cognitive science, biology, ethics, and philosophy and integrates them in the computer science approach to machine design.

Robots are defined as machines collecting information about their environment via sensors and using it to follow the instructions and perform their work. The term 'robot' initially stood for the futuristic vision of a mechanical man; however, the industrial robots usually have no resemblance to humanlike figures. A more precise definition of an industrial robot is that of a mechanical arm - an intelligent appliance that can augment the human abilities and contribute to industrial process automation. The Robot Institute of America (RIA) defined robots as "programmable multifunction devices designed to move material, parts, tools, or specialized devices through variable programmed motions for the performance of a variety of tasks.” (Brady, Gerhardt, \& Davidson, 2012). 
The challenge that robotics posed to AI at the dawn of two field's collaboration was the need to deal with real-world objects; AI was initially developed and applied for solving purely abstract, cognitive problems, while robots are physical objects equipped with mechanical effectors, sensors, and computers, the operation of which AI improves. Since robotics is the field of technology connecting perception to action, AI plays a central role in robotics by fostering the establishment of intelligent connections. AI initially aided robotics in terms of determining the proper types of knowledge required for any aspect of thinking, specifying the ways of its representation and use (Brady, 1985). In the 1990s, the increased interest in developing multiple autonomous mobile robots exhibiting cooperative behaviour led to further advancements of nanotechnology and micro electromechanical systems.

At present, the image of robots evolved into an electromechanical or biomechanical device (or group of devices) performing repetitive, pre-programmed tasks. Contemporary robots operate both under the direct human control and autonomously, under computer control. Robots are now extensively used in surgery, space and ocean exploration, in a variety of industrial spheres, and in many other dangerous areas where human beings cannot operate efficiently (Tirgul \& Naik, 2016).

\section{Intelligent Agents in Robotics}

An intelligent agent in robotics is the one deriving information about the environment through sensors and able to act upon it via activators by directing its activity towards achieving the set goal. To do no harm to humans, intelligent agents should also be rational, i.e., the ones that perform the right actions and act in the best way to succeed in the goal's completion. Robotic dexterity and intelligence are guaranteed by the inclusion of intelligence blocks into their design for retrieving data from external sources and processing it high-level decision-making (Tirgul \& Naik, 2016).

To date, two kinds of AI have found an application in robotics: software intelligence and hardware intelligence. Software intelligence is understood as the one provided by a computer, microprocessor, or microcontroller linked to hardware and giving instructions to it. In this case, software functions as a control block in which the sequence of actions is programmed to determine the robot's activities. The intelligence block of such a system may learn from the incoming data and adapt the programmed structure of decisions and actions in line with new information (Elleithy, 2008). Hardware intelligence in turn is encapsulated in 
the robot's circuits. Similar to humans who learn and develop by means of processing data from external sources via their nervous systems and brains, the robots equipped with hardware intelligence also process data and learn based on it (Govers, 2018).

The main feature distinguishing intelligent robots is their ability to plan actions, which presupposes choosing a sequence of actions for achieving a certain goal. While humans rarely stop and consciously plan a concrete sequence of actions to perform some complex activity due to their sophisticated intelligence, robots need to invest much effort into planning of any multi-step task. At the same time, robots have to perform the planned action with proper regard to any changes of the dynamic external environment and sometimes adjust actions to those changes (Tirgul \& Naik, 2016). Here comes the greatest pressing challenge of contemporary AI-enhanced robotics: allowing robots to act based only on the continuous sensory data retrieved from the external environment removes the computational and semantic challenges of keeping a large database of facts and planning actions. However, robots with a stimulus response system only cannot act as rational agents as they have no computational efficiency for evaluating how well their actions are targeted towards the goal's completion. Thus, more advancements in machine learning and decision-making are to come to make robots more intelligent and able for contingent planning.

\section{How Intelligent Robots Change the Contemporary Workplace}

While AI developments in robotics remain a trending topic, with vast investments provided to foster the technological progress and enhance machines' intelligence and dexterity, the community fears are elevating regarding the adverse impact of computerization on the global workforce. Such technological advancements as automated accounting, checkouts, smartphone apps tailored to numerous user needs have already take thousands of jobs, while the forthcoming developments of the Internet of Things and autopiloted vehicles may take even more. In line with leading tech experts' estimates (e.g., Stephen Hawking, Bill Gates), up to one-third of jobs will be taken by robots by 2025 , thus leaving hundreds of millions of people unemployed and without prospects (Brougham \& Haar, 2018).

Webster and Ivanov (2019) also pointed out that in the robotics-enhanced (and evolving) environment of today, people are ever less involved in numerous economic spheres, at least in the traditional sense of the word. While computerization is the greatest hazard for 
the low-skilled, manual jobs which machines with their limited intelligence can perform better and cheaper than people, sophisticated AI algorithms have also paved their way into nonmanual labor (e.g., accounting, legal analysis, education, medicine, etc.).

Labor market analysis shows that robots are substituting human workforce at such jobs, and the more people come across such intelligent machines in their daily work activities, the more realistic the threat of human obsolescence in the workplace is. Brougham and Haar (2018) conducted a study among 120 employees in New Zealand to test their perceptions of their future workplace as related to their Smart Technology, Artificial Intelligence, Robotics, and Algorithms (STARA) knowledge. Not surprisingly, high STARA awareness was positively correlated with skepticism, depression, and turnover intentions, coupled with low organizational commitment and poor job satisfaction. However, industry experts are still optimistic about the future of the human labor market by pointing out that massive industrial computerization will require more software development specialists, AI experts, engineers, and operators of the sophisticated equipment at the factory floors. Moreover, robots are unlikely to substitute people at such inherently human professions as sales, outreach, packaging, and consulting (Smith \& Anderson, 2010).

\section{Conclusion}

Robotics has witnessed exponential growth and development with the newest advances in artificial intelligence and machine learning. Today's machines still possess skills far from human intelligence, but they become more precise, more accurate, and more dynamically adaptive to external environment changes. Due to economic benefits of substituting the human labor force with automatons, numerous businesses automate their industrial processes at a massive scale, but this trend does not necessarily mean the extinction of human jobs. On the contrary, the increasing automation of the contemporary workplace requires more labor force with innovative technological skills. Thus, while the future of manual jobs is gloomy, knowledge-intensive, technological jobs represent a new career opportunity for millions of future workers.

\section{References}

Ashrafian, H. (2014). AIonAI: a humanitarian law of artificial intelligence and robotics. Science and Engineering Ethics, 21(1), 29-40. 
Brady, M. (1985). Artificial intelligence and robotics. Artificial Intelligence, 26(1), 79-121.

Brady, M., Gerhardt, L. A., \& Davidson, H. F. (2012). Robotics and artificial intelligence. New York, NY: Springer Science \& Business Media.

Brougham, D., \& Haar, J. (2018). Smart technology, artificial intelligence, robotics, and algorithms (STARA): employees' perceptions of our future workplace. Journal of Management \& Organization, 24(2), 239-257.

Cao, Y. U., Fukunaga, A. S., \& Kahng, A. (1997). Cooperative mobile robotics: antecedents and directions. Autonomous Robots, 4(1), 7-27.

Elleithy, K. (2008). Innovations and advanced techniques in systems, computing sciences and software engineering. New York, NY: Springer Science \& Business Media.

Frey, C. B., \& Osborne, M. A. (2013). The future of employment: how susceptible are jobs to computerization? Retrieved from http://www.oxfordmartin.ox.ac.uk/downloads/academic/The_Future_of_Employment. pdf.

Lynch, C. (2015). Stephen Hawking on the future of capitalism and inequality. Retrieved from http://www.counterpunch.org/2015/10/15/stephen-hawkings-on-the-tuture-ofcapitalism-and-inequality/

Ghahramani, Z. (2015). Probabilistic machine learning and artificial intelligence. Nature, 521, 452-459.

Govers, F. X. (2018). Artificial intelligence for politics: build intelligent robots that perform human tasks using AI techniques. Birmingham, the UK: Packt Publishing.

Smith, A., \& Anderson, J. (2010). AI, robotics, and the future of jobs. FURMAN. Retrieved from http://www.fusbp.com/wp-content/uploads/2010/07/AI-and-Robotics-Impact-onFuture-Pew-Survey.pdf

Tirgul, C. S., \& Naik, M. R. (2016). Artificial intelligence and robotics. International Journal of Advanced Research in Computer Engineering and Technology, 5(6), 1787-1793. 